\title{
Recurrence Relations for Single and Product Moments of Dual Generalized Order Statistics from a General Class of Distributions
}

\author{
Jagdish Saran* \\ Department of Statistics, University of Delhi \\ Delhi-110007, India \\ jagdish_saran52@yahoo.co.in \\ Narinder Pushkarna \\ Department of Statistics, University of Delhi \\ Delhi-110007, India \\ drpushkarna@hotmail.com \\ Rashmi Tiwari \\ Department of Statistics, University of Delhi \\ Delhi-110007, India \\ rashmistats@gmail.com \\ Received 30 August 2014 \\ Accepted 4 November 2014
}

\begin{abstract}
In this paper we derive some general recurrence relations between single and product moments of dual generalized order statistics from a general class of distributions, thus generalizing and unifying the earlier results in this direction due to several authors.

Keywords: Order Statistics; Single moments; Product moments; Recurrence relations; Generalized order statistics; Dual generalized order statistics; Power function distribution; Inverse Weibull distribution; Frechet distribution.
\end{abstract}

2010 Mathematics Subject Classification: 62G30, 62E10

\section{Introduction}

Kamps (1995a) has introduced generalized order statistics as a unified approach to several models of ascendingly ordered random variables, e.g., ordinary order statistics, sequential order statistics, progressively type II censored order statistics, upper records and upper Pfeifer records (see Kamps, 1995b or Cramer and Kamps, 2001). However, random variables that are descendingly ordered can not be integrated into this framework. Burkschat et al. (2003) introduced dual generalized order statistics (dgos) as a unification of several models of descendingly ordered random variables like,

*Corresponding author 
reversed order statistics $X_{n: n}, \ldots, X_{1: n}$, lower k-records and lower Pfeifer records. For any result in the initial model of generalized order statistics, there exists a corresponding one in the dual model. Several authors like Ahsanullah (2004), Barakat and El-Adll (2009), Arslan (2010), Jaheen and Al Harbi(2011) and Saran and Pandey (2012) have worked on dgos.

Let $\left\{X_{n}, n \geq 1\right\}$ be a sequence of independent and identically distributed random variables with cdf $F(x)$ and pdf $f(x)$. Let $X_{j: n}$ denote the $j^{t h}$ order statistic of a sample $\left(X_{1}, X_{2}, \ldots, X_{n}\right)$. Assume that $n \in N, k \geq 1, m \in R$ be the parameters such that $\gamma_{r}=k+n-r+M_{r}>0, M_{r}=\sum_{j=r}^{n-1} m_{j} \forall 1 \leq r \leq n$. By the dual generalized order statistics from an absolutely continuous distribution $F(x)$ with density function $f(x)$ we mean random variables $X^{\prime}(1, n, \tilde{m}, k), \ldots, X^{\prime}(n, n, \tilde{m}, k)$ having the joint density function of the form

$$
k\left(\prod_{j=1}^{n-1} \gamma_{j}\right)\left(\prod_{i=1}^{n-1}\left(F\left(x_{i}\right)\right)^{m_{i}} f\left(x_{i}\right)\right)\left(F\left(x_{n}\right)\right)^{k-1} f\left(x_{n}\right)
$$

for $F^{-1}(1)>x_{1} \geq x_{2} \geq \ldots \geq x_{n}>F^{-1}(0)$.

For convenience, let us define $X^{\prime}(0, n, \tilde{m}, k)=0$. It may be mentioned that for $m_{1}=\cdots=m_{n-1}=$ $0, k=1$, i.e., $\gamma_{j}=n-j+1 ; 1 \leq j \leq n-1$, the dual generalized order statistics coincide in the distribution theoretical sense with reversed order statistics. Similarly, for $m_{1}=\ldots=m_{n-1}=-1, k \in$ $N$, i.e., $\gamma_{j}=k ; 1 \leq j \leq n-1$, the joint distribution of dual generalized order statistics equals that of the lower k-records.

We may consider two cases:

Case I: $m_{1}=m_{2}=\ldots=m_{n-1}=m$.

Case II: $\gamma_{i} \neq \gamma_{j}, i \neq j, i, j=1,2, \ldots, n-1$.

For Case I, the $r^{\text {th }}$ dual generalized order statistic will be denoted by $X^{\prime}(r, n, m, k)$, and the pdf of $X^{\prime}(r, n, m, k)$ is given by

$$
f^{X^{\prime}(r, n, m, k)}(x)=\frac{c_{r-1}}{(r-1) !}[F(x)]^{\gamma_{r}-1} f(x) g_{m}^{r-1}[F(x)], \quad x \in R .
$$

Also, the joint pdf of $X^{\prime}(r, n, m, k)$ and $X^{\prime}(s, n, m, k), 1 \leq r<s \leq n$, is given by

$$
\begin{array}{r}
f^{X^{\prime}(r, n, m, k), X^{\prime}(s, n, m, k)}(x, y)=\frac{c_{s-1}}{(r-1) !(s-r-1) !}[F(x)]^{m} f(x) g_{m}^{r-1}[F(x)] \\
.\left[h_{m}(F(y))-h_{m}(F(x))\right]^{s-r-1}[F(y)]^{\gamma_{s}-1} f(y), \quad x>y,
\end{array}
$$

where

$$
\begin{aligned}
& g_{m}(x)=h_{m}(x)-h_{m}(1), \quad x \in[0,1), \\
& h_{m}(x)=\left\{\begin{array}{lll}
-\frac{1}{m+1} x^{m+1}, & \text { if } & m \neq-1 \\
-\log x, & \text { if } & m=-1 .
\end{array}\right.
\end{aligned}
$$

Let $\mu_{(r, n, m, k)}^{(i)}$ denote the $i^{t h}$ moment of the $r^{\text {th }}$ dual generalized order statistic $X^{\prime}(r, n, m, k)$. Similarly, $\mu_{(r, s, n, m, k)}^{(i, j)}$ denotes the $(i, j)^{t h}$ product moment of the $r^{t h}$ and $s^{t h}$ dual generalized order statistics. 
For Case II, the $r^{\text {th }}$ dual generalized order statistic will be denoted by $X^{\prime}(r, n, \tilde{m}, k)$, and the pdf of $X^{\prime}(r, n, \tilde{m}, k)$ is given by

$$
f^{X^{\prime}(r, n, \tilde{m}, k)}(x)=c_{r-1} f(x) \sum_{i=1}^{r} a_{i}(r)[F(x)]^{\gamma_{i}-1}, \quad x \in R .
$$

Also, the joint pdf of $X^{\prime}(r, n, \tilde{m}, k)$ and $X^{\prime}(s, n, \tilde{m}, k), 1 \leq r<s \leq n$, is given by

$$
f^{X^{\prime}(r, n, \tilde{m}, k), X^{\prime}(s, n, \tilde{m}, k)}(x, y)=c_{s-1} \sum_{i=r+1}^{s} a_{i}^{(r)}(s)\left[\frac{F(y)}{F(x)}\right]^{\gamma_{i}} \sum_{i=1}^{r} a_{i}(r)(F(x))^{\gamma_{i}} \frac{f(x)}{F(x)} \frac{f(y)}{F(y)}, x>y,
$$

where

$$
\begin{array}{r}
c_{r-1}=\prod_{j=1}^{r} \gamma_{j}, \quad \gamma_{j}=k+n-j+M_{j}, \quad r=1,2, \ldots, n, \\
a_{i}(r)=\prod_{j(\neq i)=1}^{r} \frac{1}{\left(\gamma_{j}-\gamma_{i}\right)}, \quad 1 \leq i \leq r \leq n
\end{array}
$$

and

$$
a_{i}^{(r)}(s)=\prod_{j(\neq i)=r+1}^{s} \frac{1}{\left(\gamma_{j}-\gamma_{i}\right)}, \quad r+1 \leq i \leq s \leq n
$$

In addition, for $m_{i}=m_{j}=m$, it can be shown that

$$
\sum_{i=1}^{r} a_{i}(r)(F(x))^{\gamma_{i}}=\frac{(F(x))^{\gamma_{r}}}{(r-1) !} g_{m}^{r-1}(F(x))
$$

and

$$
\begin{aligned}
& \sum_{i=r+1}^{s} a_{i}^{(r)}(s)\left(\frac{F(y)}{F(x)}\right)^{\gamma_{i}}=\frac{1}{(s-r-1) !}\left(\frac{F(y)}{F(x)}\right)^{\gamma_{s}}\left(\frac{1}{F(x)}\right)^{(m+1)(s-r-1)} \\
& \cdot\left[h_{m}(F(y))-h_{m}(F(x))\right]^{s-r-1}
\end{aligned}
$$

In the above case II, let $\mu_{(r, n, \tilde{m}, k)}^{(p)}$ denote the $p^{\text {th }}$ moment of the $r^{\text {th }}$ dual generalized order statistic $X^{\prime}(r, n, \tilde{m}, k)$. Similarly, $\mu_{(r, s, n, \tilde{m}, k)}^{(p, q)}$ denotes the $(p, q)^{t h}$ product moment of the $r^{t h}$ and $s^{t h}$ dual generalized order statistics.

Burkschat et. al (2003) defined dual generalized order statistics, alternatively, as

$$
X^{\prime}(r, n, \tilde{m}, k) \sim F^{-1}\left(W_{r}\right), \quad r=1,2, \ldots, n,
$$

where $W_{r}=\prod_{j=1}^{r} B_{j}, \quad B_{j}$ being independent random variables distributed as $\beta_{1}\left(\gamma_{j}, 1\right)$ having c.d.f. $F(t)=t^{\gamma_{j}}, t \in[0,1]$.

In this paper, we derive some general recurrence relations satisfied by the single and product moments of dual generalized order statistics from a general class of distributions with p.d.f. $f(x)$ and c.d.f. $F(x)$ satisfying the characterizing differential equation: 


$$
\left(\sum_{u=0}^{w} \alpha_{u} x^{u}\right) f(x)=\left(\sum_{v=0}^{z} \beta_{v} x^{v}\right) F(x), \quad-\infty<x<\infty,
$$

where $w$ and $z$ are integers and $\alpha^{\prime s}$ and $\beta^{\prime s}$ are arbitrary real constants.

\section{Recurrence Relations for Single Moments}

In this section, we shall establish recurrence relations for single moments of dual generalized order statistics from a general class of distributions satisfying the relation given in equation (1.8).

Case I: $m_{1}=m_{2}=\ldots=m_{n-1}=m$.

Theorem 2.1. For a positive integer $k \geq 1, n \in N, m \in Z, 1 \leq r \leq n$ and $i=0,1,2, \ldots$,

$$
\sum_{u=0}^{w} \alpha_{u} \mu_{(r, n, m, k)}^{(i+u)}=\gamma_{r} \sum_{v=0}^{z} \frac{\beta_{v}}{(i+v+1)}\left[\mu_{(r, n, m, k)}^{(i+v+1)}-\mu_{(r-1, n, m, k)}^{(i+v+1)}\right] .
$$

Proof. For $1 \leq r \leq n$ and $i=0,1,2, \ldots$, we have in view of (1.2),

$$
\sum_{u=0}^{w} \alpha_{u} \mu_{(r, n, m, k)}^{(i+u)}=\frac{c_{r-1}}{(r-1) !} \int_{-\infty}^{\infty} x^{i}[F(x)]^{\gamma_{r}-1} g_{m}^{r-1}[F(x)]\left\{\sum_{u=0}^{w} \alpha_{u} x^{u} f(x)\right\} d x .
$$

Utilizing Eq. (1.8), we get

$$
\sum_{u=0}^{w} \alpha_{u} \mu_{(r, n, m, k)}^{(i+u)}=\frac{c_{r-1}}{(r-1) !} \sum_{v=0}^{z} \beta_{v} \int_{-\infty}^{\infty} x^{i+v}[F(x)]^{\gamma_{r}} g_{m}^{r-1}[F(x)] d x .
$$

Integrating by parts of equation (2.2), treating $x^{i+v}$ for integration and rest of the integrand for differentiation, we get

$$
\begin{array}{r}
\sum_{u=0}^{w} \alpha_{u} \mu_{(r, n, m, k)}^{(i+u)}=\frac{c_{r-1}}{(r-1) !} \sum_{v=0}^{z} \frac{\beta_{v}}{(i+v+1)}\left[\gamma_{r} \int_{-\infty}^{\infty} x^{i+v+1}[F(x)]^{\gamma_{r-1}} g_{m}^{r-1}[F(x)] f(x) d x\right. \\
\left.-(r-1) \int_{-\infty}^{\infty} x^{i+v+1}[F(x)]^{\gamma_{r-1}-1} g_{m}^{r-2}[F(x)] f(x) d x\right] .
\end{array}
$$

Simplifying above terms and rearranging them, we derive the relation in (2.1) on using (1.2).

Remark 2.1. For $r=1$, the relation in (2.1) involves a term $\mu_{(0, n, m, k)}^{(i+v+1)}$ whose value will be taken as zero since we have defined $X^{\prime}(0, n, m, k)=0$.

Remark 2.2. Under the assumptions of Theorem 2.1 , with $k=1, m=0$ we shall obtain the recurrence relation for single moments of reversed order statistics from the general class of distributions (1.8).

Remark 2.3. Putting $k=0, m=-1$ in Theorem 2.1, we obtain the recurrence relation for single moments of lower record values from the general class of distributions (1.8).

Case II: $\gamma_{i} \neq \gamma_{j}, i \neq j, i, j=1,2, \ldots, n-1$. 
Theorem 2.2. For $n \in N, 1 \leq r \leq n, k \geq 1$ and $p=0,1,2, \ldots$,

$$
\sum_{u=0}^{w} \alpha_{u} \mu_{(r, n, \tilde{m}, k)}^{(p+u)}=\gamma_{r} \sum_{v=0}^{z} \frac{\beta_{v}}{p+v+1}\left[\mu_{(r, n, \tilde{m}, k)}^{(p+v+1)}-\mu_{(r-1, n, \tilde{m}, k)}^{(p+v+1)}\right] .
$$

Proof. For $1 \leq r \leq n$ and $p=0,1,2, \ldots$, we have from (1.2),

$$
\sum_{u=0}^{w} \alpha_{u} \mu_{(r, n, \tilde{m}, k)}^{(p+u)}=c_{r-1} \int_{-\infty}^{\infty} x^{p} \sum_{i=1}^{r} a_{i}(r)[F(x)]^{\gamma_{i}-1}\left\{\sum_{u=0}^{w} \alpha_{u} x^{u} f(x)\right\} d x
$$

which on using (1.8), gives

$$
\sum_{u=0}^{w} \alpha_{u} \mu_{(r, n, \tilde{m}, k)}^{(p+u)}=c_{r-1} \sum_{v=0}^{z} \beta_{v} \int_{-\infty}^{\infty} x^{p+v} \sum_{i=1}^{r} a_{i}(r)[F(x)]^{\gamma_{i}} d x .
$$

Integrating by parts treating $x^{p+v}$ for integration and rest of the integrand for differentiation, we get the required result (2.3).

Remark 2.4. For $r=1$, from the relation in eq. (2.3) we get the term $\mu_{(0, n, \tilde{m}, k)}^{(i+v+1)}$, the value of which will be zero as we have defined $X^{\prime}(0, n, \tilde{m}, k)=0$.

Remark 2.5. Putting $m_{i}=m_{j}=m$ in eq. (1.4) and using eq. (1.6), the recurrence relation for single moments of dual generalized order statistics from the general class of distributions (1.8) for Case I, i.e., when $m_{1}=m_{2}=\ldots=m_{n-1}=m$, as obtained in Theorem 2.1, can be deduced from Theorem 2.2 with $\tilde{m}$ replaced by $m$.

\section{Recurrence Relations for Product Moments}

Case I: $m_{1}=m_{2}=\ldots=m_{n-1}=m$.

Theorem 3.1. For a positive integer $k \geq 1, n \in N, m \in Z, 1 \leq r \leq s-2<n$ and $i, j=0,1,2, \ldots$,

$$
\sum_{u=0}^{w} \alpha_{u} \mu_{(r, s, n, m, k)}^{(i, j+u)}=\gamma_{s} \sum_{v=0}^{z} \frac{\beta_{v}}{(j+v+1)}\left[\mu_{(r, s, n, m, k)}^{(i, j+v+1)}-\mu_{(r, s-1, n, m, k)}^{(i, j+v+1)}\right]
$$

and for $s=r+1$,

$$
\sum_{u=0}^{w} \alpha_{u} \mu_{(r, r+1, n, m, k)}^{(i, j+u)}=\gamma_{r+1} \sum_{v=0}^{z} \frac{\beta_{v}}{(j+v+1)}\left[\mu_{(r, r+1, n, m, k)}^{(i, j+v+1)}-\mu_{(r, n, m, k)}^{(i+j+v+1)}\right] .
$$

Proof. On employing (1.3) we have, for $1 \leq r \leq s<n$ and $i, j=0,1,2, \ldots$

$$
\sum_{u=0}^{w} \alpha_{u} \mu_{(r, s, n, m, k)}^{(i, j+u)}=\frac{c_{s-1}}{(r-1) !(s-r-1) !} \int_{-\infty}^{\infty} x^{i}[F(x)]^{m} f(x) g_{m}^{r-1}[F(x)] I(x) d x,
$$

where

$$
I(x)=\int_{x}^{\infty} y^{j}\left[h_{m}(F(y))-h_{m}(F(x))\right]^{s-r-1}[F(y)]^{\gamma_{s}-1}\left\{\sum_{u=0}^{w} \alpha_{u} y^{u} f(y)\right\} d y
$$


Utilizing Eq. (1.8), we get

$$
I(x)=\sum_{v=0}^{z} \beta_{v} \int_{x}^{\infty} y^{j+v}\left[h_{m}(F(y))-h_{m}(F(x))\right]^{s-r-1}[F(y)]^{\gamma_{s}} d y .
$$

Integrating by parts treating $y^{j+v}$ for integration and rest of the integrand for differentiation and substituting the value of $I(x)$ in (3.3) and simplifying, we get the required result (3.1). In a similar manner, (3.2) can be easily established.

Remark 3.1. Under the assumptions of Theorem 3.1, with $k=1, m=0$ we shall obtain the recurrence relations for product moments of reversed order statistics from the general class of distributions (1.8).

Remark 3.2. Putting $k=0, m=-1$ in Theorem 3.1, we obtain the recurrence relations for product moments of lower record values from the general class of distributions (1.8).

Case II: $\gamma_{i} \neq \gamma_{j}, i \neq j, i, j=1,2, \ldots, n-1$.

Theorem 3.2. For $n \in N, 1 \leq r<s \leq n, k \geq 1$ and $p, q=0,1,2, \ldots$,

$$
\sum_{u=0}^{w} \alpha_{u} \mu_{(r, s, n, \tilde{m}, k)}^{(p, q+u)}=\gamma_{s} \sum_{v=0}^{z} \frac{\beta_{v}}{(q+v+1)}\left[\mu_{(r, s, n, \tilde{m}, k)}^{(p, q+v+1)}-\mu_{(r, s-1, n, \tilde{m}, k)}^{(p, q+v+1)}\right]
$$

Proof. For $1 \leq r<s \leq n$ and $p, q=0,1,2, \ldots$, we have from (1.5),

$$
\sum_{u=0}^{w} \alpha_{u} \mu_{(r, s, n, \tilde{m}, k)}^{(p, q+u)}=c_{s-1} \int_{-\infty}^{\infty} x^{p}\left\{\sum_{i=1}^{r} a_{i}(r)(F(x))^{\gamma_{i}}\right\} \frac{f(x)}{F(x)} I(x) d x
$$

where

$$
I(x)=\int_{x}^{\infty} y^{q}\left\{\sum_{i=r+1}^{s} a_{i}^{(r)}(s)\left(\frac{F(y)}{F(x)}\right)^{\gamma_{i}}\right\}\left\{\sum_{u=0}^{w} \alpha_{u} y^{u} f(y)\right\} \frac{1}{F(y)} d y .
$$

Utilizing Eq. (1.8), we get

$$
I(x)=\sum_{v=0}^{z} \beta_{v} \int_{x}^{\infty} y^{q+v}\left\{\sum_{i=r+1}^{s} a_{i}^{(r)}(s)\left(\frac{F(y)}{F(x)}\right)^{\gamma_{i}}\right\} d y .
$$

Integrating by parts treating $y^{q+v}$ for integration and rest of the integrand for differentiation, we get

$$
I(x)=\sum_{j=0}^{q} \frac{\beta_{j}}{(q+v+1)} \int_{x}^{\infty} y^{q+v+1} \sum_{i=r+1}^{s} a_{i}^{(r)}(s) \gamma_{i}\left(\frac{F(y)}{F(x)}\right)^{\gamma_{i}-1} f(y) d y .
$$

Substituting the above expression for $I(x)$ in (3.6) and simplifying, we derive the relation in (3.5).

Remark 3.3. Putting $m_{i}=m_{j}=m$ in (1.5) and using (1.7), the recurrence relation for product moments of generalized order statistics from the general class of distributions (1.8) for Case I, i.e., when $m_{1}=m_{2}=\ldots=m_{n-1}=m$ as obtained in Theorem 3.1 can be deduced from Theorem 3.2 with $\tilde{m}$ replaced by $m$. 


\section{Remark 3.4.}

$$
\text { Setting } \alpha_{u}=\left\{\begin{array}{lll}
\frac{1}{p}, & \text { if } & u=1 \\
0, & \text { if } & u \neq 1
\end{array}\right.
$$

and

$$
\beta_{v}=\left\{\begin{array}{lll}
1, & \text { if } & v=1 \\
0, & \text { if } & v \neq 1
\end{array}\right.
$$

we observe that (1.8) reduces to

$$
\frac{x}{p} f(x)=F(x),
$$

which is the characterizing differential equation for power function distribution with p.d.f. in the form

$$
f(x)=p v^{-p} x^{p-1} \quad, \quad 0<x \leq v, v>0 .
$$

One can deduce the recurrence relations for single and product moments of dual generalized order statistics from power function distribution for the particular values of parameters involved.

\section{Remark 3.5.}

$$
\text { Setting } \alpha_{u}=\left\{\begin{array}{lll}
\frac{1}{p \theta^{p}}, & \text { if } & u=p+1 \\
0, & \text { if } & u \neq p+1
\end{array}\right.
$$

and

$$
\beta_{v}=\left\{\begin{array}{lll}
1, & \text { if } & v=1 \\
0, & \text { if } & v \neq 1
\end{array}\right.
$$

we observe that (1.8) reduces to

$$
\frac{f(x)}{p \theta^{p}} x^{p+1}=F(x),
$$

which is the characterizing differential equation for inverse Weibull distribution with p.d.f. in the form

$$
f(x)=p \theta^{p} x^{-(p+1)} e^{-\left(\frac{\theta}{x}\right)^{p}}, \quad x>0, p, \theta>0 .
$$

One can deduce the recurrence relations for single and product moments of dual generalized order statistics from inverse Weibull distribution for the particular values of parameters. 


\section{Remark 3.6.}

$$
\text { Setting } \alpha_{u}=\left\{\begin{array}{lll}
\frac{\lambda^{\theta}}{\theta}, & \text { if } & u=0 \\
0, & \text { if } & u \neq 0
\end{array}\right.
$$

and

$$
\beta_{v}= \begin{cases}1, & \text { if } \quad v=1 \\ 0, & \text { if } \quad v \neq 1\end{cases}
$$

we observe that (1.8) reduces to

$$
\frac{\lambda^{\theta}}{\theta} f(x)=x^{-(\theta-1)} F(x),
$$

which is the characterizing differential equation for two parameter Frechet distribution with p.d.f. in the form

$$
f(x)=\left(\frac{x}{\lambda}\right)^{-\theta-1}\left(\frac{\theta}{\lambda}\right) \exp \left\{-\left(\frac{x}{\lambda}\right)^{-\theta}\right\}, \quad x>0, \quad \theta>0, \quad \lambda>0 .
$$

One can deduce the recurrence relations of single and product moments of dual generalized order statistics from Frechet distribution for the particular values of parameters.

\section{References}

[1] M. Ahsanullah, A Characterization of the uniform distribution by dual generalized order statistics, Comm. Statist. Theory Methods, 33 (2004) 2921- 2928.

[2] G. Arslan, On a characterization of the uniform distribution by generalized order statistics, J. Comput. Appl. Math., 235(16) (2010) 4532-4536.

[3] H.M. Barakat and M.E. El-Adll, Asymptotic theory of extreme dual generalized order statistics, Stat. Probabil. Lett., 79 (2009) 1252-1259.

[4] M. Burkschat, E. Games and U. Kamps, Dual generalized order statistics, Metron, LXI(1)(2003) 13-26.

[5] E. Cramer and U. Kamps, Sequential k-out-of-n systems. In N. Balakrishnan and C. R. Rao, eds., Handbook of Statistics - Advances in Reliability, Vol. 20 (2001) 301-372. Elsevier, Amsterdam.

[6] Z.F. Jaheen and M.M. Al Harbi, Bayesian Estimation Based on Dual Generalized Order Statistics from the Exponentiated Weibull Model, J. Stat. Theory Appl., 10(4) (2011) 591-602.

[7] U. Kamps, A concept of generalized order statistics, ( B.G. Teubner Stuttgart, 1995a).

[8] U. Kamps, A concept of generalized order statistics, J. Statist. Plann. Inference, 48 (1995b) 1-23.

[9] U. Kamps and E. Cramer, On distribution of generalized order statistics, Statistics, 35 (2001) 269-280.

[10] J. Saran and A. Pandey, Recurrence relations for marginal and joint moment generating functions of dual generated order statistics from power function distribution, Pak. J. Statist, 28(2) (2012) 231-238. 